\title{
Ultrastructural and Immunofluorescence Studies of Placental Tissue in Hypertensive Diseases of Pregnancy
}

\author{
${ }^{1}$ John Paul Judson, ${ }^{3}$ Lee Pui Fun, ${ }^{1}$ Vishna Devi Nadarajah, ${ }^{4}$ Sivalingam Nalliah, \\ ${ }^{2}$ Srikumar Chakravarthi, ${ }^{2} \mathrm{P}$. Thanikhacalam and ${ }^{5} \mathrm{~L}$. Santhanaraj \\ ${ }^{1}$ Department of Human Biology, ${ }^{2}$ Department of Pathology, \\ Faculty of Medicine, International Medical University, Malaysia \\ ${ }^{3}$ Research Laboratory, Department of Postgraduate Studies, \\ International Medical University, Malaysia \\ ${ }^{4}$ Department of Obstetrics and Gynaecology, Clinical School, \\ International Medical University, Malaysia \\ ${ }^{5}$ Electron Microscopy Unit, Institute of Medical Research, Malaysia
}

\begin{abstract}
Gestational hypertension and preeclampsia account for 5-7\% of the maternal mortality and morbidity worldwide. Gestational hypertension and preeclampsia is characterised by blood pressure of $140 / 90 \mathrm{~mm} \mathrm{Hg}$ or greater after the 20 th week of gestation. The aetiology of gestational hypertension and preeclampsia is closely related to the disorder of placenta implantation. It is thought that shallow trophoblast invasion into maternal decidua causes malfunction of the utero-placental arteries, thus leading to both the diseases. The exact cause of shallow trophoblast invasion remains controversial. Placenta implantation involves activation and migration of trophoblast into the decidua and myometrium. These processes are dependent on keratin expression where activation of appropriate adhesion molecules and integrins are essential for appropriate trophoblast activity. This integrated study aimed to detect and analyse the trophoblastic keratin expression, as well as to examine the placenta ultrastructure for presence of degenerative changes in normal, gestational hypertensive and preeclamptic placenta. Immunofluorescence double staining method was performed on placental sections and trophoblastic expression of keratin 19 (K19) and keratin 18 (K18) were analysed qualitatively and quantitatively in normal $(n=10)$, gestational hypertensive $(n=10)$ and preeclamptic $(n=10)$ placenta. Statistical analysis of means was performed using one-way ANOVA. Representative placental samples from each of three groups were analysed via electron microscopy. Immunofluorescence results showed that both K19 and 18 expression in gestational hypertension and preeclampsia were reduced compared to normal but significant reduction was observed only for K19 expression in gestational hypertensive placenta. Gestational hypertensive placenta showed more reduction than preeclampsia for both keratins. Electron microscopic results demonstrated obvious degeneration and significant changes in the placental ultrastructure in all representative samples of gestational hypertension and preeclampsia when compared to normal. The redearchers conclude that the fundamental link exists between keratin expression and placental ultrastructural changes. The present keratin findings, especially in gestational hypertension, need to be confirmed by larger studies in future.
\end{abstract}

Key words: Placenta, preeclampsia, keratin, trophoblast, pregnancy, diseases, Malaysia

\section{INTRODUCTION}

Hypertensive disorders complicate $6-8 \%$ of pregnancies worldwide, they are the second leading cause of maternal mortality after embolism in the United States of America (Yucesoy et al., 2005). In Malaysia, pregnancy-induced hypertension (now termed as gestational hypertension) and preeclampsia are common antenatal complications seen in about $6 \%$ of obstetric patients admitted to hospitals and hypertensive diseases of pregnancy accounted for $14.1 \%$ of total maternal deaths between the years 1997 and 2000 (Myatt, 2002). Hypertensive disorders of pregnancy are also associated with increased risk of maternal-perinatal adverse outcomes. Some of the severe maternal complications include seizures, intracerebral haemorrhage, pulmonary

Corresponding Author: John Paul Judson, Department of Human Biology, Faculty of Medicine, International Medical University, 57000 Kuala Lumpur, Malaysia 
oedema, acute renal failure, liver dysfunction and coagulopathy. Fetal complications include abruptio placentae, Intrauterine Growth Retardation (IUGR), premature delivery and intrauterine death (Hubel, 1999).

The exact aetiology of gestational hypertension, preeclampsia and eclampsia remains unknown to this day. Nevertheless, the placenta is firmly believed to play a primary role in the aetiology of the disease because symptoms usually resolve spontaneously after delivery (Piering, 1993). Based on literature, a two-staged immunological aetiology has been proposed. The first stage is the relative failure of early trophoblast invasion and remodelling of spiral arteries lead to abnormal placentation, a rather common finding in preeclamptic pregnancies.

This leads to poor blood supply to the placenta, exposing it to ischaemia and oxidative stress. The second stage is characterised by a generalised maternal inflammatory response evoked by the placenta, involving the activation of leukocytes and endothelial dysfunction (Shembrey and Noble, 1995). There are evidences that in preeclampsia, there is increased placental apoptosis (Zhou et al., 1997) and placental oxidative stress leads to an increased shedding of apoptotic and/or syncytiotrophoblast debris into the maternal circulation (McMaster et al., 2004). Such trophoblastic debris interacts with maternal leukocytes and endothelial cells, stimulating the release of pro-inflammatory cytokines which could trigger the maternal disease (Sargent et al., 2003). It is well established that while the shedding of trophoblastic debris and fragments into maternal blood does occur even in healthy pregnancy, this is increased in preeclampsia (Chua et al., 1991).

The human placental trophoblast has been reported to express keratins in developmental, differentiative and functional specific patterns. Prior to that, studies have confirmed that keratins $\mathrm{K} 8,18$ and 19 were present in trophoblast cells and were maintained through differentiation into various subsets of trophoblast (Ockleford et al., 1993) and a number of trophoblastic studies used either anti-pankeratin antibodies (Ockleford et al., 2004; Tempfer et al., 2000) or known specific anti-keratin antibodies (Hefler et al., 2001).

Using immunofluorescence confocal laser scanning microscopy and immunoelectron microscopy, it has been described at least five keratin proteins are present in villous trophoblast and the same five in extravillous trophoblast with the remaining 14 keratins tested undetectable in these tissues. Four out of five of the keratins detected $(K 7,8,18$ and 19) were found to be downregulated in preeclamptic chorionic villous trophoblast compared to the healthy ones and the difference was statistically significant. These results further support the findings of earlier studies
(Ockleford et al., 2004) whereby placental trophoblastic keratin expression was found to be down regulated in preeclampsia. Hence, Ockleford and Ahenkorah suggested that the villous trophoblast in preeclamptic placentae is cytoskeletally weaker with respect to the filaments made from these specific keratin proteins and it could be one reason why trophoblast is deported in greater quantity in preeclampsia. Therefore, there is a need to examine for placental damage, especially for degenerative and apoptotic changes at the endothelial and villous levels by electron microscopy that might not be evident in gross and light microscopic studies.

Prior to the study above, there were other studies which discovered a link between keratins and preeclampsia. Plasma keratin concentrations in preeclamptic women were markedly increased compared to the healthy pregnant women (Schrocksnadel et al., 1992). Since then, there have been a number of studies associating preeclampsia with increased plasma levels and placental expression of keratins, specifically K18 and 19 (Tempfer et al., 2000; Hefler et al., 2001; Ahenkorah et al., 2008). Hence, it was intriguing when Ockleford and Ahenkorah discovered that keratins were downregulated in preeclamptic placenta trophoblast cells. This warrants for more research to investigate the relation between keratins and preeclampsia. Few studies in the past have examined the ultrastructural aspects of preeclamptic placenta (De Luca Brunori et al., 2005). Despite the studies describing evidence of altered trophoblastic keratin expression and placenta ultrastructural changes in preeclampsia, all were independent of each other and did not include gestational hypertension in their study. Thus, it is imperative to investigate both findings within the same population and to include gestational hypertension as an entity.

Recent evidence suggests that examining the alterations in placental keratin expression and its effect on the placental ultrastructure of normotensive, preeclamptic and gestational hypertensive mothers will allow better understanding about the aetiology of the disease.

The objectives of this integrated study were to study the cytoskeletal architecture by detecting the presence and analysing specific keratins in placenta of normal, gestational hypertensive and preeclamptic mothers by immunofluorescence; as well as to study the ultrastructure of the placenta in the three groups and examine for any degenerative and apoptotic changes that might be the cause for triggering the disease process.

\section{MATERIALS AND METHODS}

Patient recruitment and tissue sampling: Approval for the study was obtained from International Medical 
University (IMU) Joint-Committee of the Research Committee and the Ethics Committee (ID No.: BMS I02/2008 (10) and also from Clinical Research Centre (CRC) of Ministry of Health Malaysia (ID No.: NMRR-08-15663112). Pregnant women with gestational ages from $20-40$ weeks were recruited from Antenatal Screening Room at Hospital Tuanku Ja'afar, Seremban, Malaysia between January 2009 and May 2009. Three groups of participants were recruited: participants with healthy pregnancy (normotensive) $(\mathrm{n}=10)$, participants with gestational hypertension ( $\mathrm{n}=10)$ and lastly, participants with preeclampsia $(\mathrm{n}=10)$. The diagnoses of gestational hypertension (de novo hypertension after 20 weeks of gestation) or preeclampsia (de novo hypertension after 20 weeks of gestation together with proteinuria $=$ $300 \mathrm{mg} / 24 \mathrm{~h}$ urine or other signs/symptoms) were made based on Malaysia's Clinical Practice Guidelines on Management of Hypertension which follows the classification by the Australasian Society for the Study of Hypertension in Pregnancy (ASSHP) and is endorsed by International Society for the Study of Hypertension in Pregnancy (ISSHP) (Bancher-Todesca et al., 2001). Pregnant women with chronic hypertension, pre-existing renal disease, gestational diabetes mellitus, multiple gestation (e.g., twin pregnancy) and smokers were excluded from the study. Written informed consent was obtained from each participant. Relevant clinical data of each participant were then obtained from the maternal antenatal records and delivery records.

Placental samples were taken following vaginal or caesarean delivery. A random site on the placentae was chosen and a section of placental tissue $\left(1 \mathrm{~cm}^{2}\right.$ surface area through whole placental thickness) was obtained. Peripheral areas of the placentae were avoided to ensure all specimens obtained were of similar thickness. A small piece of roughly $1 \mathrm{~mm}^{3}$ was then cut from the section and immersed in $2.5 \%$ glutaraldehyde in $0.1 \mathrm{M}$ phosphate buffer at $2-8^{\circ} \mathrm{C}$ for electron microscopic studies. The remainder of the section was fixed in $10 \%$ neutral buffered formalin at room temperature and later processed and paraffin-embedded at histopathology laboratory for immunofluorescence studies.

Immunofluorescence technique and fluorescent microscopy: Paraffin-embedded placental specimens were cut to produce sections at $4 \mu \mathrm{m}$ thickness. For this study, indirect immunofluorescence double staining method was employed in order to make comparison between the distribution patterns of 2 proteins, keratin 18 (K18) and keratin 19 (K19) in the placenta trophoblastic cells lining the villi. Multiple staining methods, such as double staining in this study, allows comparison between two or more proteins to be made within the same slide preparation from the same specimen block (Bataille et al., 2006). Before staining was carried out, optimisation step of antibody dilution was performed to obtain the optimum antibody concentration to be used.

Antibodies were diluted with ready-to-use antibody diluent (Dako REAL ${ }^{\text {TM }}$ Antibody Diluent, S2022). Primary antibodies monoclonal mouse anti-human cytokeratin 19 (Clone RCK 108, Dako Cytomation, M0888) optimum dilution was 1:80 while monoclonal mouse anti-human cytokeratin 18 (Clone DC 10, Dako Cytomation, M7010) dilution was 1:40. Secondary antibodies FITC-conjugated polyclonal rabbit anti-mouse (Dako Cytomation, F0261) and TRITC-conjugated polyclonal rabbit anti-mouse (Dako Cytomation, R0270) were both used at 1:20 dilution. All antibodies were stored and used as per manufacturer's protocol.

Sections were dewaxed, rehydrated in ethanol series and treated with heat-induced epitope antigen retrieval method (Dako Target Retrieval Solution, S2367) as per manufacturer's protocol. Following two washes of 2-min phosphate buffered saline with $0.05 \%$ Tween 20 (PBS-T), sections were incubated with first primary antibodies mouse anti-human $\mathrm{K} 19$ for $1 \mathrm{~h}$ at room temperature after which sections were washed with three changes of 4-min PBS-T. The sections were then incubated with first secondary antibodies FITC-conjugated rabbit anti-mouse for $30 \mathrm{~min}$ at room temperature in the dark, followed by three washes of $4 \mathrm{~min}$ PBS-T. Subsequently sections were incubated with second primary antibodies mouse antihuman $\mathrm{K} 18$ for $1 \mathrm{~h}$ at room temperature in the dark (to preserve FITC signals). Similarly, sections were washed with three changes of $4 \mathrm{~min}$ PBS-T and lastly, the sections were incubated with second secondary antibodies TRITC-conjugated rabbit anti-mouse for $30 \mathrm{~min}$ at room temperature in the dark. Sections were washed with three changes of $4 \mathrm{~min}$ PBS-T and anti-fade mounting medium (Dako Fluorescence Mounting Medium, S3023) was applied before applying glass coverslips. Sections were observed under Nikon Fluorescent Microscope using standard fluorescent filters. Digital images were captured and analysed qualitatively and quantitatively using NIS elements for basic research software.

\section{Quantitative fluorescent measurement and statistical} analysis: Quantitative analysis of fluorescent-stained sections was done by measuring the area percentage of high-intensity fluorescent pixels (Ockleford et al., 2004; Ahenkorah et al., 2008). Firstly, the section of interest was divided into four imaginary quadrants and a field view was chosen randomly from each of the four quadrants. Under $400 \mathrm{x}$ magnification, two images were captured for 
each field view, one for visualisation of FITC signals (representing K19) and one for TRITC signals (representing K18). A total of four FITC images and four TRITC images were captured for each placental tissue section. Using the pixel classifier function of the software, the area percentage of high-intensity fluorescent pixels (FITC intensity $\geq 25$ units, TRITC intensity $\geq 20$ units) in each image was measured.

All values were recorded in Excel spreadsheet and statistical analysis was done using SPSS 15.0 for Windows. For comparisons between mean values of the different groups the parametric one-way Analysis of Variance (ANOVA) test was used to examine significance. The data was analysed at 95\% confidence interval and a p-value of $<0.05$ was considered significant.

Electron microscopy: Glutaraldehyde fixed specimens were transferred to Electron Microscopy Unit in Institute of Medical Research (IMR), Kuala Lumpur, Malaysia and were processed according to the IMR Electron Microscopy Unit's Protocol.

The specimens were taken out of glutaraldehyde, washed with six changes of phosphate buffer and then post-fixed in $1 \%$ osmium tetraoxide for $1 \mathrm{~h}$ before being washed with six changes of phosphate buffer again. En bloc staining of the specimens with $2 \%$ uranyl acetate for 5 min was performed in the dark to enhance contrast.

Specimens were then washed with distilled water and dehydrated in ethanol series of $70 \%$ ethanol, $95 \%$ ethanol and finally two changed of $100 \%$ ethanol. Specimens were then immersed in a mixture of liquid plastic resin and propylene oxide (a transition solvent) to allow the plastic to gradually infiltrate into the tissue cells. Following that the specimens were transferred to pure resin and incubated in embedding oven at $60^{\circ} \mathrm{C}$ for overnight to allow polymerisation. Semi-thin sections of $0.5 \mu \mathrm{m}$ were produced first using an ultramicrotome and stained with toluidine blue to be observed under light microscope. Finally, ultra-thin sections of $90 \mathrm{~nm}$ were cut from the chosen blocks and were stained with uranyl acetate followed by lead citrate. The sections were then viewed under the Tecnai G2 Transmission Electron Microscope (TEM) and analysed qualitatively.

\section{RESULTS AND DISCUSSION}

Immunofluorescence study-qualitative analysis of trophoblastic K19 and 18 expression: Under the highest magnification possible by the Nikon microscope $(1000 \mathrm{x})$, it appeared that both K19 and 18 stained the same trophoblastic area within the same placental section. There was no difference in the pattern of expression between the two keratins in all the 30 placental sections when superimposition of K19 image on 18 image was done. Both K19 image and 18 image were captured under the same microscope field view.

In all 30 immunofluorescence stained placental specimens, it was observed that the trophoblastic lining of the chorionic villi were labelled positively with the antiK19 and anti-18 antibodies. Villous stroma and fetal capillary endothelial cells did not stain for both keratins (Fig. 1). In certain specimens whereby basal plate was sampled and hence available for analysis the extravillous trophoblast also stained positively for both K19 and 18 (Fig. 2).

Immunofluorescence study-quantitative analysis of trophoblastic K19 and K18 expression: Based on the data obtained by the immunofluorescence quantitative method the mean area percentage of high intensity FITC pixels (representing levels of keratin 19 expression) observed for normotensive placenta $(\mathrm{n}=10$ ) was the highest among three groups at 14.15 (Fig. 3). For gestational hypertensive placenta $(n=10)$ it was lower at 7.88 and for preeclamptic

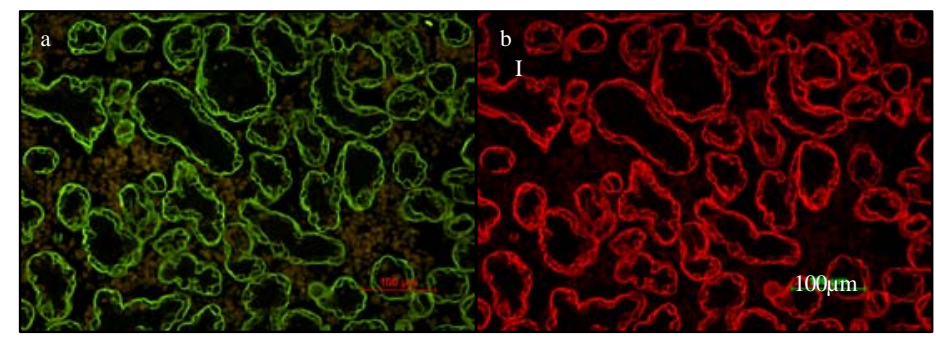

Fig. 1: Keratin as a trophoblast marker. In both pictures a and $b$ the anti-keratin antibodies stained only the trophoblast cells lining the villi. Villous stroma and fetal capillary endothelium were not stained. Both images were taken from the same section under the same field view. a: K19 labelled with FITC; b: K18 labelled with TRITC. Dull yellow spots shown in Figure are red blood cells. Microscopic magnification at $200 \mathrm{x}$ 


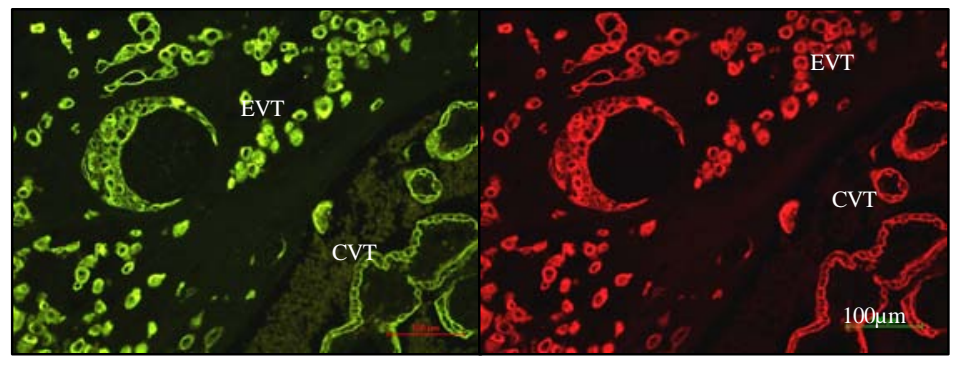

Fig. 2: Expression of K19 and 18 in Extravillous Trophoblastic (EVT) cells. On the lower right corners of both figures are chorionic villous trophoblasts (CVT). Microscopic magnification at 200x

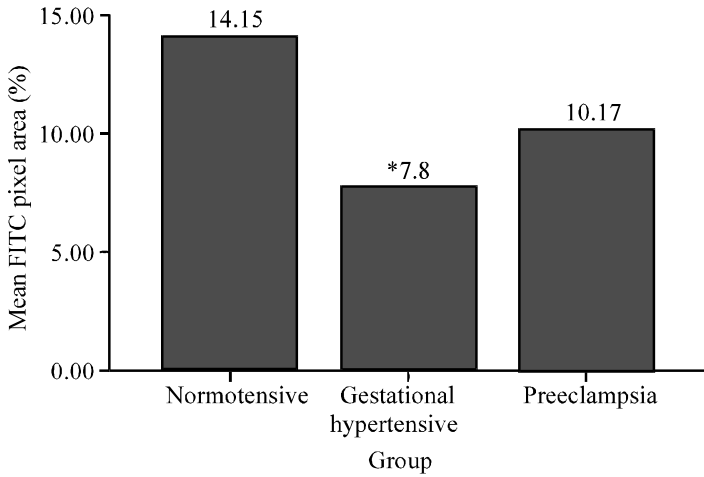

Fig. 3: Mean area percentage of FITC fluorescing pixels representing levels of K19 expression in normotensive, gestational hypertensive and preeclamptic placenta. Statistical analysis revealed that level of K19 expression in gestational hypertensive (marked*) differed significantly from normotensive placenta at $\mathrm{p}<0.05$. No significant difference was observed between normotensive and preeclampsia

placenta $(n=10)$ it was 10.17 . It was interesting to note that preeclamptic placenta exhibit a higher value than gestational hypertensive placenta. The results of a statistical test (ANOVA) showed a significant difference between the three groups $(\mathrm{p}=0.022)$. Post hoc test (Tukey) revealed that there was a significant difference between normotensive placenta and gestational hypertensive placenta at $\mathrm{p}=0.017$. There was no significant difference between normotensive and preeclamptic placenta and also between gestational hypertensive and preeclamptic placenta.

The mean area percentage of high intensity TRITC pixels (representing levels of keratin 18 expression) observed for normotensive placenta $(\mathrm{n}=10)$ was the highest among three groups at 10.48 (Fig. 4) while gestational hypertensive placenta $(\mathrm{n}=10)$ showed a lowest value of 6.71 . The value observed for preeclamptic

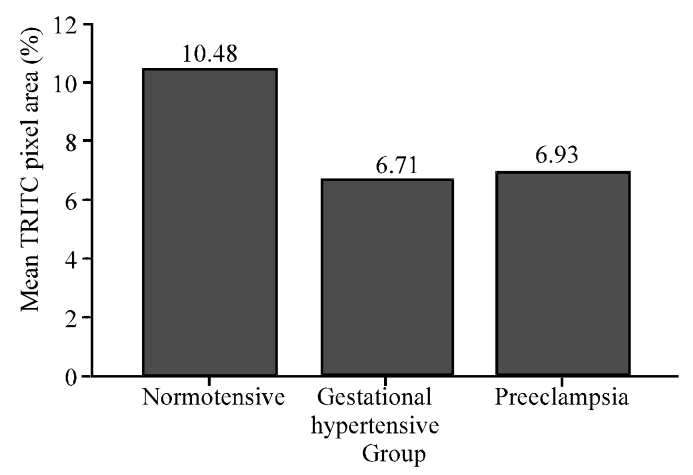

Fig. 4: Mean area percentage of TRITC fluorescing pixels representing levels of K18 expression in normotensive, gestational hypertensive and preeclamptic placenta. Statistical analysis revealed that there was no significant difference between three groups

placenta $(n=10)$ was 6.93 which interestingly was lower than normotensive but higher than gestational hypertensive placenta. The trend of keratin 18 expression between three groups was similar to that of keratin 19 . However, the differences between the groups for keratin 18 expression were not significant $(\mathrm{p}=0.088)$.

Three representative samples from each group of normotensive, gestational hypertensive and preeclamptic placenta were used for the transmission electron microscopic study. The examination of the chorionic villi from full term normotensive placenta shows typical structure in which the syncytiotrophoblast appeared as a thin layer of syncytium. The fetal capillaries which about the syncytial layer were dilated with thin walls and the endothelial cells were flattened. Basement membrane can be seen between the syncytial layer and the endothelial lining of fetal capillary. Microvilli (Fig. 5) were found on the border of the syncytium facing the lacunae (fetalmaternal interface). High magnifications of the endothelial 
cells showed presence of very fine electron-lucent intracellular filaments (Fig. 6). The examination of ultrastructure in gestational hypertensive and preeclamptic placenta reveals marked morphological damage compared to the normotensive placenta. The chorionic villi in these diseased placenta showed, in some areas, syncytiotrophoblast with reduced and deformed microvilli (Fig. 5-7).

There were increased vacuolation and swollen mitochondria which are signs of apoptosis and cell degeneration (Fig. 6 and 7). The thickness of basement membranes showed a certain degree of variations in the

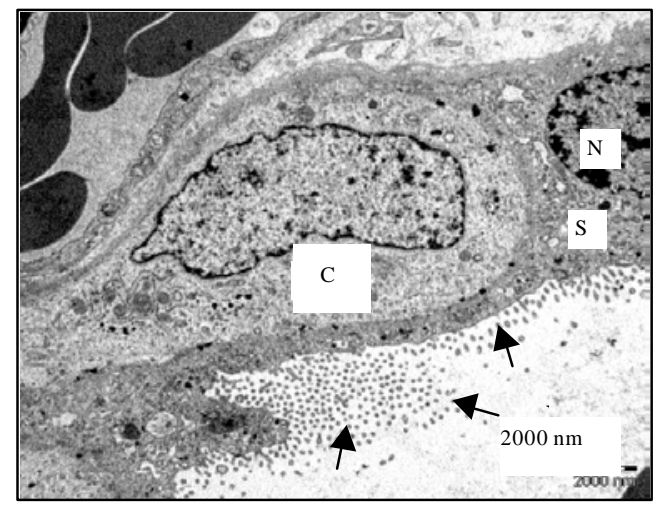

Fig 5: Electron microphotograph of a portion of villus from full-term normotensive placenta showing the syncytial layer $(\mathrm{S})$ and part of its nucleus $(\mathrm{N})$ with microvilli (arrows), a less-dense cytotrophoblast (C) just within the syncytium. L- lacunae

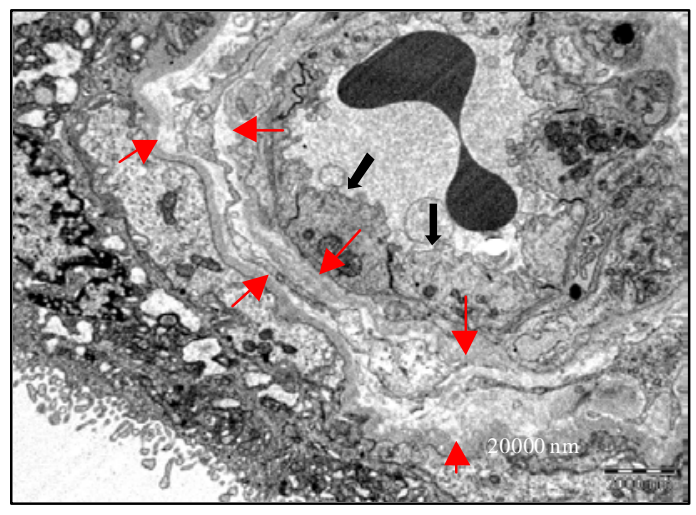

Fig. 6: Electron microphotograph of swollen capillary endothelium in gestational hypertensive placenta. It shows grossly swollen and ragged endothelial lining (black arrows) and there is increased gap between the capillary and the overlying trophoblast layer with reduced extrcellular matrix (red arrows) placenta examined. In some areas, the endothelial cells lining the fetal capillary were swollen and bulbous as well as ragged. It was noticed that the endothelium in the preeclamptic specimens exhibits more electron-dense intracellular filaments compared to the normotensive specimens (Fig. 7 and 8). In the past, the keratin findings and alterations in placental ultrastructure were seen mainly in preeclampsia and were mostly independent studies done on Western populations (DiFederico et al., 1999; Chambers et al., 2001; Ishihara et al., 2002). This study is unique as looks at placenta from 3 groups of pregnant women in an integrated manner (through immunofluorescence and electron microscopic studies). It is also at the forefront of establishing a basic link

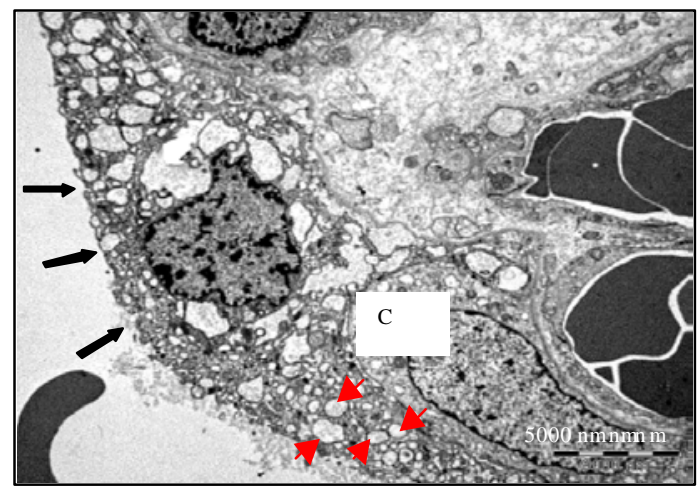

Fig. 7: Electron microphotograph of a portion of villus from full-term normotensive placenta showing the syncytial layer (S) and part of its nucleus $(\mathrm{N})$ with microvilli (arrows), a less-dense cytotrophoblast (C) just within the syncytium. L- lacunae

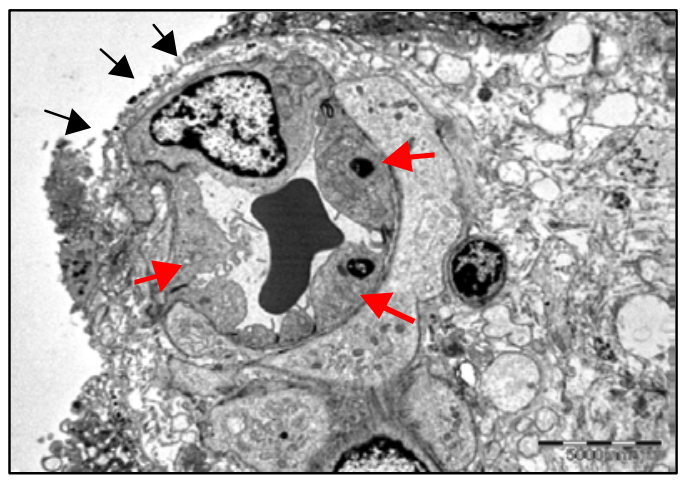

Fig. 8: Electron microphotograph of portion of a villus from preeclamptic placenta. Notice the grossly deformed capillary and markedly swollen endothelial cells (red arrows). The syncytial covering (black arrows) is reduced with loss of microvilli, leaving only debris-like layer 
between the two findings with pregnant women in a Malaysian community. The result of the study confirms that keratins are specific trophoblast cell markers, as reported by earlier studies (Allaire et al., 2000; Leung et al., 2001). As shown in Fig. 2 and 3 the K19 and 18 antibodies only stained the extravillous trophoblasts and the trophoblastic lining of the chorionic villi. The villous stroma and fetal capillary endothelium were not stained. The rationale behind using two closely related keratins, K19 and 18, in this study is because different studies reported varying results for their expression in placental tissue. In year 2008 , it was reported that four keratins $(\mathrm{K} 7,18,19$ and 20) were downregulated in preeclampsia. In year 2000 and 2001, placental expressions of K19 and K18 have been studied independently in their relation to preeclampsia (Lyall and Greer, 1996; Holthe et al., 2004).

However, both studies reported increased keratin expression in preeclampsia. Hence, we decided to investigate the levels of placental expression of these two keratins in Malaysian community, in contrast to the earlier studies which were done on Western populations.

Comparison of placental keratins between normal and preeclamptic placenta have been reported beginning in late 20th century. As mentioned earlier, placental expression of K19 and 18 were thought to increase in preeclampsia in studies conducted in year 2000 and 2001, respectively (Lyall and Greer, 1996; Holthe et al., 2004). However, the grading method employed in both studies was rather subjective, in which they grade strong and widespread staining as positive and weak staining as negative. Then in year 2004, Ockleford et al. (2004) used a confocal laser scanning microscope, a sophisticated tool to analyse and compare the overall keratin expression in normal and preeclamptic placenta (Dekker and Sibai, 1998). They found that pankeratin expression in preeclampsia to be significantly reduced when measuring the area percentage of high-intensity fluorescing pixels. Subsequently, it was discovered that four keratins, including K19 and 18 were significantly downregulated in preeclampsia. They then suggested that the trophoblast cells in preeclampsia is cytoskeletally weaker and hence are deported in greater quantities. In all the studies above, none included gestational hypertension as an entity to be compared with normal and preeclampsia.

The lack of data on gestational hypertension was the main reason it was included in this preliminary study. This allows determination of any variation between gestational hypertension and preeclampsia if present. It is of great interest to compare these two because there were cases of gestational hypertension progressing to preeclampsia while $\mathrm{BP}$ of some patients returned to normal after delivery without developing proteinuria (Roberts et al., 2003; Fisher, 2004). Findings in this study may form the basis for future study to investigate the reason why some cases of gestational hypertension progress to preeclampsia while some cases recovered uneventfully. For this study, comparison of placental K19 and K18 expression between normotensive, gestational hypertensive and preeclamptic placenta yielded interesting results. A similar trend was found for both K19 and 18 expression whereby both were reduced in gestational hypertension and preeclampsia when compared to normal (Ahenkorah et al., 2008; Roberts and Hubel, 2009).

A key finding in this study is that more reduction was observed in gestational hypertension than preeclampsia for both $\mathrm{K} 19$ and 18 and the reduction was significant for $\mathrm{K} 19$ in gestational hypertension when compared to normal. This is an interesting observation since gestational hypertension is traditionally known as being less severe clinically compared to preeclampsia. The fact that the reduction in keratin expression was not significant in preeclampsia indicates the possibility that keratins do not play a major role in the apparent increased apoptosis and shedding of syncytiotrophoblast seen in preeclampsia. Preeclampsia has long been implicated with multifactorial pathogenesis, with the intrinsic factors (genetic) interacting with the environmental factors (diet, diabetes, obesity) (Roberts and Hubel, 2009).

The most recent review of the two-staged model of preeclampsia in year 2009 explained that reduced placental perfusion (stage 1) is not sufficient to cause preeclampsia (stage 2) but requires the interaction of maternal constitutional factors (genetic, behavioural and environmental). The review also described there are more than one linking factors (oxidative stress, syncytiotrophoblast microparticles and anti-angiogenic factors) between the two stages and no one common factor is seen in all preeclamptic pregnancies.

Moreover, it is also believed that in preeclampsia, there is increased trophoblastic turnover rate (as a compensatory mechanism against injury) which indicates augmented trophoblast proliferation together with apoptosis and deportation (Redman and Sargent, 2003). Hence, the increased trophoblast proliferation might have masked the effect of keratin reduction in preeclampsia. This explains why the keratin reduction in preeclampsia is not significant but in gestational hypertension the reduction is significant. The significant difference seen in K18 expression between all three groups could be explained by the fact that the level of $\mathrm{K} 18$ expression was 
represented by TRITC (secondary antibody to K18 primary antibody). This is because TRITC (red fluorescence) which represented K18 expression is less intense against a dark background compared to FITC (green fluorescence) which represented K19 expression. The microscope software (NIS Elements BR) might not have been sensitive enough to detect the reduction in $\mathrm{K} 18$ which binds to TRITC. In addition, the antibody dilution range given by the manufacturer (DAKO) for K19 and 18 is different. Due to this reason, quantitative comparison of level of expression between K19 and 18 were not made possible. As stated earlier, there is evidence of increased trophoblastic turnover rate in preeclampsia. The increased proliferation and apoptosis leads to greater number of syncytial fragments being shed into the maternal blood. Biochemically, it has been proven that there are significantly increased amounts of subcellular syncytiotrophoblast microparticles in the maternal circulation.

Ultrastructurally, it has been shown via electron microscopy that there is severe alteration in preeclampsia when compared to normal (Sargent et al., 2003). The electron microscopic analysis of our study demonstrated obvious ultrastructural changes in gestational hypertensive and preeclamptic placenta specimens compared to the normal placenta specimens (De Luca Brunori et al., 2005). In comparison, the study went a step further by including gestational hypertension as an entity as well. Other than the apoptotic signs seen in the syncytial layer, there was an apparent increase in the density of the ndothelial intracellular filaments in preeclamptic placenta, a finding similar to that of De Luca Brunori et al. (2005). However, no interendothelial junction alteration or breakage which was reported by Brunori was seen in this study. No syncytial fragments were seen in the maternal lacunae as well.

This could be due to the lack of sampling area as only $1 \mathrm{~mm}^{3}$ of specimen was taken from each placenta. Electron microscopic processing of multiple samples from the same placenta was not feasible owing to the cost limitations.

Nonetheless, the observation of increased signs of apoptosis and cell damage in the syncytial layer is in line with the concept that there is more syncytial debris shed into maternal lacunae in preeclampsia (Redman and Sargent, 2003), as apoptosis and cell damage preceeds the shedding of syncytial fragments into maternal lacunae. Correlation analysis between placental ultrastructural changes and keratin findings was not possible as only representative samples from each of the three groups were processed for electron microscopy because of cost limitations. Even so the fact that ultrastructural alterations were seen in all representative samples of gestational hypertensive and preeclamptic placenta taken from the same population from which the keratin findings were obtained, supports that there is a connection between keratin expression and trophoblast deportation.

The interesting discovery of the variation in keratin expression between gestational hypertension and preeclampsia should also prompt future studies to include gestational hypertension as an entity to be compared to preeclampsia. The development of a scheme to grade and compare the ultrastructural changes in gestational hypertension and preeclampsia will enhance the accuracy of the results and their extrapolation. Lastly, it would be fascinating to explore further the role of endothelial intracellular filaments by using quantitative electron microscopy. The difference in electron density of these filaments seen in normal, gestational hypertension and preeclampsia can be studied quantitatively and any association with the disease could provide a whole new insight to the possible mechanisms of the disease.

\section{CONCLUSION}

In this study, the findings of the preliminary study showed that placental K19 expression is reduced significantly in gestational hypertension compared to normal. It can also be confirmed that there are obvious ultrastructural changes in gestational hypertensive and preeclamptic placenta and that a fundamental link exists between keratin expression and placenta ultrastructural alterations.

\section{ACKNOWLEDGEMENT}

This research was funded by research grant BMS I02/2 (12) from the International Medical University, Kuala Lumpur, Malaysia.

\section{REFERENCES}

Ahenkorah, J., S. Byrne, P. Bosio and C.D. Ockleford, 2008. Immunofluorescence confocal laser scanning microscopy and immuno-electron microscopic identification of keratins in human maternofetal interaction zone. J. Cellular Mol. Med., 13: 735-748.

Allaire, A.D., K.A. Ballenger, S.R. Wells, M.J. McMahon and B.A. Lessey, 2000. Placental apoptosis in preeclampsia. Obstet. Gynecol., 96: 271-276.

Bancher-Todesca, D., L. Hefler, H. Zeisler, C. Schatten, P. Husslein and H.G. Placental, 2001. Expression of Cytokeratin 18 and serum levels of tissue polypeptide antigen in women with pregnancyinduced hypertension. Hypertension Pregnancy, 20: 89-98. 
Bataille, F.M.D., S. Troppmann, F.M.D. Klebl, G.M.D. Rogler, B.P. Stoelcker and F.M.D. Hofstadter, 2006. Multiparameter immunofluorescence on paraffin-embedded tissue sections. Applied Immunohistochemistry Mol. Morphol., 14: 225-228.

Chambers, J.C., L. Fusi, I.S. Malik, D.O. Haskard, M. De Swiet and J.S. Kooner, 2001. Association of maternal endothelial dysfunction with preeclampsia. J. Am. Med. Associat., 285: 1607-1612.

Chua, S., T. Wilkins, I. Sargent and C. Redman, 1991. Trophoblast deportation in pre-eclamptic pregnancy. Br. J. Obstet. Gynaecol., 98: 973-979.

De Luca Brunori, I., L. Battini, E. Brunori, P. Lenzi, A. Paparelli and M. Simonelli, 2005. Placental barrier breakage in preeclampsia: Ultrastructural evidence. Eur. J. Obstetrics Gynecol. Reproductive Biol., 118: 182-189.

Dekker, G.A. and B.M. Sibai, 1998. Etiology and pathogenesis of preeclampsia: Current concepts. Am. J. Obstetrics Gynecol., 179: 1359-1375.

DiFederico, E., O. Genbacev and S.J. Fisher, 1999. Preeclampsia is associated with widespread apoptosis of placental cytotrophoblasts within the uterine wall. Am. J. Pathol., 155: 293-301.

Fisher, S.J., 2004. The placental problem: Linking abnormal cytotrophoblast differentiation to the maternal symptoms of preeclampsia. Reprod. Biol. Endocrinol., 2: $53-53$.

Hefler, L.A., C.B. Tempfer, D. Bancher-Todesca, C. Schatten, P. Husslein and G. Heinze, 2001. Placental expression and serum levels of cytokeratin18 are increased in women with preeclampsia. J. Soc. Gynecologic Investigat., 8: 169-173.

Holthe, M.R., A.C. Staff, L.N. Berge and T. Lyberg, 2004.Leukocyte adhesion molecules and reactive oxygen species in preeclampsia. Obstetrics Gynecol., 103: $913-922$.

Hubel, C.A., 1999. Oxidative stress in the pathogenesis of preeclampsia. Proc. Soc. Exp. Biol. Med., 222: 222-235.

Ishihara, N., H. Matsuo, H. Murakoshi, J.B. LaoagFernandez, T. Samoto and T. Maruo, 2002. Increased apoptosis in the syncytiotrophoblast in human term placentas complicated by either preeclampsia or intrauterine growth retardation. Am. J. Obstetrics Gynecol., 186: 158-166.

Leung, D.N., S.C. Smith, K.F. To, D.S. Sahota and P.N. Baker, 2001. Increased placental apoptosis in pregnancies complicated by preeclampsia. Am. J. Obstetrics Gynecol., 184: 1249-1250.

Lyall, F. and I. Greer, 1996. The vascular endothelium in normal pregnancy and pre-eclampsia. Rev. Reproduct., 1: 107-116.

McMaster, M.T., Y. Zhou and S.J. Fisher, 2004. Abnormal placentation and the syndrome of preeclampsia. Seminar Nephrol., 24: 540-547.
Myatt, L., 2002. Role of placenta in preeclampsia. Endocrine, 19: 103-111.

Ockleford, C., T. Malak, A. Hubbard, K. Bracken, S.A. Burton and N. Bright, 1993. Confocal and conventional immunofluorescence and ultrastructural localisation of intracellular strength-giving components of human amniochorion. J. Anatomy, 183: 483-505.

Ockleford, C.D., S. Byrne, R. Sanders and P. Bosio, 2004. Confocal laser scanning microscope study of cytokeratin immunofluorescence differences between villous and extravillous trophoblast: Cytokeratin downregulation in pre-eclampsia. Microscopy Res. Technique, 64: 43-53.

Piering, W.F., J.G. Garancis, C.G. Becker, J.A. Beres and J. Lemann, 1993. Preeclampsia related to a functioning extrauterine placenta: Report of a case and 25-year follow-up. Am. J. Kidney Dis., 21: $310-313$.

Redman, C.W.G. and I.L. Sargent, 2003. Pre-eclampsia, the placenta and the maternal systemic inflammatory response: A review. Placenta, 24: 21-27.

Roberts, J.M. and C.A. Hubel, 2009. The two stage model of preeclampsia: Variations on the theme. Placenta, 30: $32-37$.

Roberts, J.M., G. Pearson, J. Cutler and M. Lindheimer, 2003. Summary of the NHLBI working group on research on hypertension during pregnancy. Hypertension, 41: 437-445.

Sargent, I.L., S.J. Germain, G.P. Sacks, S. Kumar and C.W. Redman, 2003. Trophoblast deportation and the maternal inflammatory response in pre-eclampsia. J. Reprod. Immunol., 59: 153-160.

Schrocksnadel, H., G. Daxenbichler, E. Artner, G. SteckelBerger and O. Dapunt, 1992. Tissue polypeptide antigen and pre-eclampsia. Lancet, 340: 53-54.

Shembrey, M.A. and A.D. Noble, 1995. An instructive case of abdominal pregnancy. Aust. N. Z. J. Obstet. Gynaecol., 35: 220-221.

Tempfer, C.B., D. Bancher-Todesca, H. Zeisler, C. Schatten, P. Husslein and A.R. Gregg, 2000. Placental expression and serum concentrations of cytokeratin 19 in preeclampsia. Obstet. Gynecol., 95: 677-682.

Yucesoy, G., S. Ozkan, H. Bodur, T. Tan, E. Caliskan and B. Vural, 2005. Maternal and perinatal outcome in pregnancies complicated with hypertensive disorder of pregnancy: A seven year experience of a tertiary care center. Arch. Gynecol. Obstet., 273: 43-49.

Zhou, Y., C.H. Damsky and S.J. Fisher, 1997. Preeclampsia is associated with failure of human cytotrophoblasts to mimic a vascular adhesion phenotype. One cause of defective endovascular invasion in this syndrome?. J. Clin. Investigat., 99: 2152-2164. 